\title{
Sociología de
}

\section{la infancia y política social: ¿Compatibilidades posibles? ${ }^{1}$}

\author{
René Unda Lara ${ }^{2}$
}

\section{Síntesis}

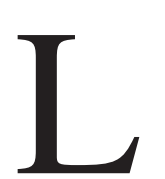

a posibilidad de que las políticas sociales, en el capitalismo global, tengan eficacias que no sólo se limiten a compensar parcialmente a los eufemísticamente llamados sectores vulnerables de la sociedad, entre ellos, la infancia, se afinca, desde el ámbito de lo académico y específicamente universitario, en la producción de una socio-lógica de la infancia; un desafío que supone continuidades en la reflexión sociológica pero, sobre todo, rupturas respecto de aproximaciones y enfoques que han encapsulado la niñez, aislándola del conjunto de relaciones sociales mediante la difuminación de representaciones de infancia comúnmente aceptadas, o a través de tendencias más vinculadas a ingenierías sociales que han aplicado directa e indiscriminadamente principios sociológicos a las problemáticas de infancia y adolescencia haciendo abstracción de que estos objetos condicionan, en sí mismo, la producción de una específica y particular sociología.

Esta producción sería una de las condiciones de redefinición de indicaciones de política; constituiría, en suma, la esencial contribución académica para repensar la política social, como espacio de agregación de sentidos con potencialidad transformadora, y las mismas políticas sociales dirigidas a infancia en un momento histórico en el que la política social ha dejado de cumplir funciones de regulación de la política económica y ha sido supeditada y funcionalizada a esta. En el marco

\footnotetext{
${ }^{1}$ Versión sustentada en la ponencia presentada en el XXIV Congreso de la Asociación Latinoamericana de Sociología (ALAS). Arequipa, Nov. 2003.

${ }^{2}$ Director de la Maestría en Política Social para Promoción de la Infancia y Adolescencia de la Universidad Politécnica Salesiana, Sede Quito.
} 
general de las profundas transformaciones del Estado y de la relación Estado-Sociedad, la búsqueda de compatibilidades entre el nivel de una sociología de la infancia y el de las políticas sociales de infancia representa no solo la posibilidad de redefiniciones teóricas y conceptuales de las políticas sociales sino también nuevas posibilidades para la misma producción de politicidad en general.

\section{Entrada}

¿Por qué preguntarse si una sociología de la infancia y las políticas sociales pueden ser compatibles? ¿Qué sentido tiene activar interrogantes de este tipo y qué posibles consecuencias teóricas tiene para la política social, para el campo de articulaciones entre sociología de la infancia y políticas sociales y para la misma sociología de la infancia?

Desde estas preguntas se despliega, dentro de los límites de este trabajo, los ejes de análisis que consideramos pertinentes para comprender y cuestionar la articulación entre sociología y políticas sociales dirigidas a infancia y adolescencia.

Dichos ejes se condensan en una breve revisión de las principales dificultades y desafíos para la constitución de una sociología de la infancia, seguida de una muy elemental aproximación a los tipos de discursividades con las que se aborda el tema desde perspectivas sociológicas y, finalmente, un análisis introductorio al campo de política social visto desde una doble vertiente conceptual: como posibilidad de construcción de nuevas relaciones entre Estado, sociedad y mercado, y como prescripciones paliativas dirigidas a atenuar los efectos de las políticas económicas, que ha sido la tendencia dominante en la mayor parte de los Estados nacionales subordinados política y económicamente a los centros de decisión y, más actualmente, a las indicaciones y presiones de fuerzas corporativas anónimas.

Ciertamente, hablar de la existencia de una sociología de la infancia como un campo sociológico constituido, plantea más dudas que certezas por varias razones que serán expuestas en el desarrollo de este trabajo. En este sentido, se intenta articular algunos presupuestos conceptuales orientados hacia una posible delimitación inicial de una sociología de la infancia con el difuso campo de las políticas sociales. 
Esta articulación -sociología de la infancia y políticas sociales de infancia- que podría aparecer como obvia, ya que la infancia ha sido tradicionalmente objeto de las llamadas políticas y programas sociales como producto de una indiferenciación entre lo que es la infancia como hecho social y lo que es la infancia como categoría analítica y hecho sociológico, es puesta en cuestión y examinada con una intencionalidad claramente teórica. Se trata, en suma, de contribuir a la superación de una mera perspectiva instrumental y aplicada con la que, generalmente, la sociología ha abordado el campo de infancia y adolescencia.

Para tal efecto, analizaremos, por una parte, algunos de los supuestos planteados en torno de la sociología de la infancia, reconociendo, en primer término, varias de las dificultades presentes en el tratamiento de infancia y, por otra, retomando la discusión sobre política social. Quedan, finalmente, planteadas algunas argumentaciones desde las que con más cautela que audacia se sostiene que la sociología de la infancia podría contribuir a repensar la producción de Política Social, entendida como un campo de agregaciones en el que las desbordadas e incontenibles fuerzas del mercado puedan supeditarse a renovadas lógicas de organización social.

\section{Algunos problemas metodológicos}

Pensar una sociología de la infancia, que vaya más allá de la directa aplicación de principios sociológicos generales a las problemáticas de infancia, como condición de la producción de políticas sociales para este sector poblacional específico, representa un complejo desafío en varios sentidos.

Primero, un mínimo estatuto teórico de una sociología de la infancia supone la revisión del acumulado sobre el tema, lo cual en sí mismo constituye una tarea nada exenta de dificultades, tanto por la dispersión de enfoques con las que se ha abordado la problemática como por la relativa ausencia de una producción sociológica sostenida.

Luego, el campo de infancia y adolescencia se encuentra muy atravesado por una vasta gama de representaciones sociales ancladas en el sentido común e instaladas en el cotidiano individual y colectivo, hecho que también supone más dificultades que posibilidades analíticas y explicativas sobre el tema. De modo adicional, la concepción de las 
políticas sociales, fundamentada históricamente en una razón asistencial y su articulación con "los sectores más vulnerables" de la sociedad, configuran un escenario complejo para una tarea como la propuesta.

Este trabajo, que se inscribe en un programa de investigación más amplio y cuyo espacio natural es el de las maestrías en derechos de la infancia y adolescencia que llevan a cabo varias universidades de la subregión andina con el apoyo de Save the Children Suecia, tiene un doble propósito: 1) plantear vías analíticas y explicativas del fenómeno infancia como objeto sociológico con sus propias particularidades, relaciones y autonomía relativa, y 2) analizar críticamente el campo de articulaciones entre políticas sociales e infancia como espacio privilegiado de aplicaciones sociológicas, cuando no de proyectos basados en representaciones instaladas en el sentido común y que aparecen como "obvias" en gran parte de las prácticas y discursos institucionales. Sobre la base de este objetivo se intentará dejar establecidos algunos lineamientos que permitan pensar en recomposiciones en las indicaciones de política social más que en sus reingenierías, a partir de lo que mínimamente debería considerar una sociología de la infancia.

Para tales efectos, se desarrolla un análisis de las posibilidades de aproximación sociológica al campo de infancia y adolescencia, desde aquellas discursividades que enmarcan la niñez y el niño como objetos desprovistos de una determinada condición social, ajenos a las relaciones sociales, institucionales y familiares, hasta concepciones y formulaciones con las que se abriría la posibilidad de construir una sociología de la infancia. Evidentemente, caben en una posición intermedia, dentro de estas posibilidades, los enfoques aplicativos de la sociología y las ingenierías sociales, expresados, sobre todo, en las políticas y programas sectoriales para infancia y adolescencia.

El recorrido analítico en el campo de las políticas sociales, que en este trabajo constituye más bien un ejercicio de síntesis, se centra en el interés por reconocer algunos presupuestos sociológicos en la genealogía de la producción de políticas sociales más que en el balance y evaluación de las mismas; esto es, privilegiar el marco de relaciones de producción de politicidad, constituido y delimitado por el Estado, sus tipologías y su contraparte societal, así como por sus espacios de articulación, por sobre la interpretación de los efectos y resultados de la aplicación de políticas y programas. 
Cabe indicar, que la misma naturaleza de la discusión sobre políticas sociales conduce a referencias muy específicas -la concepción y tipos de política social así como las variantes de política social entre uno y otro estado latinoamericano son elementos nada desdeñables para el análisis- y que, en el presente caso, situarán como eje referencial al Estado ecuatoriano en distintos períodos históricos.

Un mínimo ordenamiento metodológico, creemos, exige partir de un somero análisis de los discursos con los que se ha abordado el campo de la infancia, los aportes y dificultades que estas perspectivas han generado para lo que podría ser el establecimiento de un estatuto teórico mínimo para una sociología de la infancia.

Este inicial ejercicio se articulará con las concepciones y usos de política social dirigida a infancia, en referencia directa al caso ecuatoriano, con el objeto de demostrar, dentro de los límites de este trabajo, la necesidad de pensar una sociología de la infancia que no solo trascienda los discursos y lugares comunes en torno a infancia sino que, ante todo, permita comprenderla como un objeto sociológico particular en el conjunto de relaciones y articulaciones sociales en general. Todo esto, con una finalidad práctica muy concreta: contribuir a la redefinición de las indicaciones de política y sus subsiguientes procesos como posibilidad reconstitutiva del Estado en tiempos de repliegue estatal frente a la acometida de dinámicas -transnacionales- corportativistas y de descentramiento de la política como espacio ordenador de sociedad.

\section{Despejando el camino: de las dificultades a los desafíos}

Si bien el análisis explicativo por niveles de los diversos discursos en torno a la infancia, desarrollado por autores como J. Sánchez Parga o Ferrán Casas, resulta fundamental para el esclarecimiento del conjunto de las representaciones sociales de la infancia. En este trabajo se adopta una vía analítica orientada hacia su tratamiento como tipos de discurso sobre infancia, ya que en todos ellos están presentes, aunque de modo muy distinto y disperso, elementos conceptuales de la sociología. Se trata, en primer término, de identificar las procedencias y enfoques desde las que se aborda el campo de la infancia, partiendo de la compleja pregunta, sociológicamente hablando, sobre ¿qué es el niño? 
De modo previo, sin embargo, existe, ciertamente, una primera gran dificultad: cómo entendemos el proceso de construcción sociológica de un determinado objeto en tiempos de gran dispersión discursiva, fragmentación de sentido y multivocidad posmoderna. No daremos demasiados rodeos -no más de los indispensables- sobre la definición de un objeto sociológico, es decir, de cómo el hecho social ("prostitución infantil", por ejemplo) se convierte en hecho sociológico o realidad pensada desde un conjunto de causas específicas y en transformación (determinaciones-condicionamientos estructurales y relaciones-acciones particulares del problema de la prostitución infantil) mediante la activación categorial de interrogantes, es decir, mediante la investigación.

Una siguiente consideración de partida consiste en el reconocimiento de que la misma producción sociológica se ha diversificado y especializado a tales niveles y con arreglo a fines de reproducción social, que muchos de sus campos particulares han sido tratados como meros objetos de intervención más que como objetos teóricos. Evidentemente, detrás de estos tratamientos existe un complejo entramado de interacciones que van desde las determinaciones más globales en términos económicos y políticos hasta los intereses particulares de corporaciones, también constitutivas de la lógica de lo global.

En suma, más que el interés por el desarrollo de un pensamiento sociológico, lo que se ha evidenciado, como tendencia general, es una amplificación e intensificación de la presencia y utilización de las ingenierías sociales diseñadas para subsanar el problema infancia, hecho que ha repercutido en la adecuada definición de ciertos presupuestos básicos para desarrollar una sociología de la infancia.

Las mencionadas dificultades se expresan en dos dimensiones: a) el cúmulo de representaciones sociales, sistemas de creencias y lugares comunes ampliamente compartidos por franjas importantes de la sociedad que ha servido para abordar discursiva y operativamente las problemáticas de infancia, y b) una notoria dispersión, tanto de sentidos como de discursos y prácticas, con la que se ha intervenido en el campo de infancia.

Lo primero tiene varios efectos. Quizás, el más relevante para desarrollar un ejercicio crítico desde una perspectiva académica sea las ingentes dosis de emocionalidad y espontaneísmo con que gran parte de la institucionalidad pública y privada ha intervenido la infancia y, específicamente, las problemáticas de los sectores más desprotegidos de la infancia. Es bien conocido que cuando se asumen las representaciones 52 
como supuestos teóricos, el extravío en laberintos discursivos es inminente y el despliegue de una serie de estrategias y recursos tiene, finalmente, muy bajas eficacias.

No se trata de desconocer la necesidad de actuar de forma urgente e inmediata sobre varias de las acuciantes problemáticas de infancia y adolescencia y, mucho menos, de menospreciar el trabajo de instituciones y personas convencidas -parafraseando a Dolto- de "la causa de los niños". Sin embargo, lo que no pueden desconocer quienes trabajan desde diversos ámbitos y niveles en el campo de infancia, es que muchos de los tratamientos, en términos de políticas sociales o programas integrales y sectoriales dirigidos a infancia, son plenamente coherentes con las lógicas asistencialitas y compensatorias que, en el caso de los Estados periféricos, son lógicas constitutivas de la espiral de empobrecimiento en la relación actual Norte-Sur ${ }^{3}$.

Adicionalmente, sobre este primer punto, la reflexión sociológica sobre la infancia debe enfrentar todo un conjunto compacto de creencias y representaciones instaladas en el sentido común y que, en lo fundamental, se condensan en percepciones que definen al niño como no apto, como adulto pequeño, o como entidad externa a las relaciones sociales en su conjunto. Así, "el trabajo sociológico de objetivación"“ en el campo de la infancia y la adolescencia resulta mucho más difícil.

La segunda observación, derivada de la anterior y relativa a la notoria dispersión en el abordaje y definición de campos y articulaciones conceptuales propios de una sociología de la infancia, no ofrece mayores obstáculos en cuanto a su constatación. Existe un importante acumulado producido sobre problemáticas de niñez en el que se ha tratado muy diversos tópicos, interesantes sí, pero desarticulados de elaboraciones teóricas propias del campo conceptual de una sociología de la

\footnotetext{
${ }^{3}$ Cabe recordar que si bien las políticas sociales han estado atravesadas históricamente por concepciones basadas en la asistencia y la compensación en cualquier modelo de Estado, en el caso de las sociedades periféricas y empobrecidas, la asistencia y compensación, a diferencia de sociedades desarrolladas, han tendido a agudizar las diferencias sociales (cfr. Montagut Teresa, Política Social, Ariel, Barcelona, 2000)

${ }^{4}$ Sánchez-Parga, J., Orfandades infantiles y adolescentes. Introducción a una sociología de la infancia. Abya-Yala, Maestría en Política Social para Promoción de la Infancia y Adolescencia, Red Latinoamericana de Maestrías Infancia, UPS, Quito, 2004.
} 
infancia. Se evidencia, pues, el problema de la indiferenciación entre el problema social y el objeto de estudio sociológico. Se interviene directamente sobre el hecho sin haberlo problematizado suficientemente.

Podría decirse, como acertadamente puntualiza la socióloga española Marta Martínez ${ }^{5}$ que lo que ha existido, en rigor, es una presencia de la infancia en la sociología y no una sociología de la infancia como un campo cultural específico. Por una parte, operan representaciones no procesadas sociológicamente y, por otra, se aplican sin mediación conceptual alguna, preceptos y principios sociológicos generales a las distintas situaciones y problemas de infancia, lo cual ha postergado e impedido la construcción de una socio-lógica de infancia.

Esta tendencia se explica, más que por una desviación facilista, por la relativa ausencia de investigaciones que definan categorialmente -sociológicamente- su objeto de estudio. Se indaga directamente el hecho social, sin que se considere necesario, teórica y metodológicamente, su problematización en términos conceptuales.

El resultado de lo anterior es, generalmente, una amalgama de conceptos de distinta naturaleza, usos indiscriminados de categorías no pertinentes o ajenas al campo investigado. Ciertamente, puede generarse productos residuales como la caracterización de una problemática, generalmente estadísticos, o, en el mejor de los casos, registros descriptivos útiles pero claramente insuficientes que aluden, simplemente, a factores estructurales como causas directas de las distintas problemáticas de la infancia. La exclusión, por ejemplo, proceso y producto del (des) orden global actual es, sin duda, un factor estructural del proceso de callejización del niño pero insuficiente como causa explicativa específica de dicho proceso.

Como puede observarse hasta aquí, las dificultades señaladas en la construcción del objeto sociológico provienen de una matriz de representaciones sociales en las que predomina la tentación de actuaciones e intervenciones directas sobre la "población objetivo". Sus efectos, no sólo determinan sucesivos postergamientos en la tarea de construir -pensar, una sociología de la infancia sino que, además, aplazan posibles

\footnotetext{
${ }^{5}$ Martínez Muñoz, M. Recensión crítica del texto de Lourdes Gaitán "El espacio social de la infancia. Los niños en el Estado del Bienestar", en NATS, Revista Internacional desde los Niños/as y Adolescentes Trabajadores, Año VI-No 10Mayo 2003.
} 
marcos de acción sostenible en favor de la infancia, cuando no agudizan o encubren varias de sus problemáticas.

Creemos que, sin mayores y precipitadas pretensiones, la construcción de una sociología de la infancia debe plantear el desarrollo de un proceso en dos sentidos de orden conceptual y metodológico. El primero, atendiendo a las formulaciones de la sociología clásica, al acumulado del conocimiento sociológico, en el que los hechos y problemas sociales son hechos producidos por la sociedad; de este enunciado se desprenden dos cuestiones fundamentales para la investigación sociológica: a) el hecho social está articulado con otros hechos sociales que operan como determinaciones, condicionamientos o efectos de aquel, y b) ningún hecho social se produce al margen de su espacio "natural" de producción: la sociedad. Por lo tanto, ningún hecho social es externalizable, ajeno y extraño a la sociedad.

El segundo, en el que los fenómenos sociales deben ser problematizados desde las nuevas y cambiantes causas que producen dichos fenómenos, produciendo, con ello, nuevas categorías. El desempleo, por ejemplo, no puede seguir siendo explicado únicamente por el crecimiento demográfico y por los límites del mercado en términos de sobreproducción o subconsumo. Han aparecido nuevos factores como el desarrollo de las nuevas tecnologías y producción intensiva, la desterritorialización de la producción, desregulación y flexibilización laboral, entre otros.

De acuerdo con esas dos orientaciones generales, las posibilidades de desarrollo progresivo de la sociología de la infancia están ancladas en las categorías de continuidad-ruptura y sus correspondientes modelos explicativos; "en el trabajo intelectual serio no hay comienzos absolutos, y se dan pocas continuidades sin fracturas" ${ }^{.}$.

Puede argüirse al respecto, que la masa crítica de la sociología de la infancia es todavía "muy precaria, incipiente, ensayística..." , lo cual es bastante cierto, sin embargo, existe un acumulado conceptual y metodológico de la sociología que permite conocer cómo se generaron y desarrollaron muchos subcampos sociológicos, cuáles fueron sus continuidades respecto de los principios sociológicos básicos, sus contradic-

\footnotetext{
${ }^{6}$ Hall Stuart, "Cultural Studies: two paradigms". Media, Culture and Society, London, 1980.

7 Sánchez-Parga, J. op. cit.
} 
ciones o tensiones internas, y cuáles son las categorías específicas que constituyen su campo conceptual particular.

Pero desde la perspectiva de las "fracturas" o rupturas epistemológicas, la construcción de una sociología de la infancia prefigura un horizonte mucho más prometedor en términos científicos y académicos. Desde la misma ubicación de la infancia como objeto sociológico, la necesidad de rupturas epistemológicas deviene inevitable.

La crítica de las representaciones sociales dominantes de infancia, el desmontaje conceptual de sistemas de creencias, y la reversión conceptual de tópicos sobre infancia, solo serán posibles si se plantean rupturas que demuestren la validez de la ciencia como referente explicativo de distintas problemáticas concretas, apelando al principio epistemológico según el cual afirma que "la ciencia sería innecesaria si la realidad se explicara por sí sola" (K. Marx). En lo que sigue, retomaremos el tema de las rupturas en el marco más delimitado y preciso de las distintas discursividades sobre infancia como una de las vías analíticas que permite abordar sociológicamente este campo.

\section{Tipos de discursividades sobre infancia}

Sobre la base de las dificultades señaladas hasta aquí, trataremos de identificar una muy elemental tipología discursiva, presente en el campo de infancia. Según Sánchez Parga, quien despliega algunas de las diferenciaciones discursivas sobre la infancia que propone Ricardo Luchini, tres serían los niveles discursivos sobre la infancia: el infantológico, la sociología aplicada, y la sociología de la infancia. Adicionalmente, este autor identifica un nivel de discurso al que denomina jurídico-tecnocrático, expresado en convenciones, declaraciones y acuerdos, códigos y normativas desde las que se han judicializado muchos de los problemas sociales de la infancia.

Más allá de situar la discusión en torno a que si son niveles o tipos de discursos sobre la infancia, lo fundamental radica en la explicación sobre el proceso de construcción de estos discursos, en sus implicaciones y efectos.

Lo que Sánchez Parga denomina “infantología” podría interpretarse, empleando la terminología de la tradición sociológica, como una aproximación sociológica "ingenua" sobre la infancia. Como se ha dicho ya, en esta perspectiva converge un cúmulo de representaciones 
sociales que por sus efectos emotivos y su carga de afectividad difícilmente son cuestionados. Es más, constituyen referentes para la acción e intervenciones sobre grupos de niños/as desde organismos e instituciones con sentido filantrópico o asistencialista, por una parte, y desde organismos que, en el otro extremo, adultizan la infancia al atribuirle características ajenas a sus reales condiciones.

Al niño/a, desde esta perspectiva, se lo concibe inconscientemente como un objeto externo a las relaciones sociales, producto de una severa dificultad para desprenderse de ideas y representaciones muy internalizadas y difundidas, a la vez que compartidas por vastos segmentos poblacionales.

Una reciente investigación sobre representaciones institucionales de la infancia llevada a cabo en Quito, da cuenta, entre otros resultados, de cómo el/la niño/a es definido/a (inocente, manipulador, tierno, cruel, sincero, desvalido, autosuficiente, etc...) por una gama diversa de profesionales de instituciones que trabajan en este campo, hecho que evidencia que en este tipo de discurso pueden operar simultáneamente tanto una idealización infantilista (del/la "niño/a ángel") como una proyección adultista (del/la niño/a "adulto pequeño").

Si las consecuencias de estas representaciones en el plano del conocimiento son obstáculos que dificultan e impiden la comprensión del/la niño/a de manera relacional (en su relación con el adulto y la sociedad en general) contribuyendo a una indeterminación conceptual de la infancia y de la diversidad de infancias, las consecuencias aplicativas son nefastas debido a que las intervenciones mediante programas y proyectos al sustentarse en estas representaciones suponen que "ayudan" a los/as niños/as reforzando cualidades circunstanciales y contingentes, pero, además, encapsulándolo o externalizándolo como entidad al margen de unas determinadas relaciones sociales, familiares, escolares.

Por ello, una primera línea de reflexión básica para la formulación de políticas sociales de la infancia es que el/la niño/a no puede ser comprendido al margen de la sociedad en la que se inscribe. Sus cualidades y características ni son solo de él ni surgen exclusivamente de él, sino que se adquieren y se (re) producen en las prácticas sociales. Si no es una entidad al margen de la sociedad, las políticas sociales y los derechos de infancia habrán de considerar que tienen que atravesar -analítica y objetivamente- varios espacios donde el/la niño/a desarrolla su socialización. 
Un segundo tipo de discurso que identifica el autor precitado, es el de la sociología aplicada a problemas de la infancia. Más allá de las dificultades señaladas en el numeral anterior, referidas al postergamiento de la elaboración y discusión teóricas, el fundamental problema radica en su estrecha articulación con la producción de políticas sociales. Y, como se verá más adelante, las políticas sociales, desde esta perspectiva, no pueden sino limitarse a sus lógicas originarias en el Estado moderno, es decir, al asistencialismo compensatorio ${ }^{8}$, a atenuar parcial y temporalmente los efectos de los desequilibrios producidos por la implantación de las políticas económicas.

Cabe dejar planteado que la asistencia, en el moderno Estado de bienestar europeo (welfare state) no necesariamente tuvo la misma connotación y efectos sociales que en el común de los Estados latinoamericanos. Quizás la mayor diferencia radicó en que el asistencialismo en el llamado Estado de bienestar de las sociedades desarrolladas estaba orientado hacia la igualación compensatoria y permanente de oportunidades y accesos a la vida social de sectores en situación desfavorable, mientras que en sociedades empobrecidas, el Estado y sus políticas asistencialistas encubren las reales causas de los problemas sociales y postergan sus soluciones debido, fundamentalmente, a factores asociados a la estructura y composición del Estado, tradicionalmente construido de espaldas a la sociedad y cooptado por intereses corporativos.

Por ello, la reflexión sobre las políticas sociales de la infancia debe pasar necesariamente por un detenido análisis de la cuestión teórica del Estado y de sus formas particulares en cada sociedad. De igual forma, la construcción de una sociología de la infancia supone la comprensión de las relaciones entre Estado, sociedad, familia, escuela y medios de comunicación para el establecimiento de un estatuto teórico básico que permita comprender la diversidad de infancias en cada tipo de sociedad.

Un tercer tipo de discurso, y es el que tratamos de construirlo, es el de la sociología de la infancia; aquel que presenta al/la niño/ña como un hecho socialmente producido, pero que no se limita a la utilización de categorías generales para posibles explicaciones sino que con-

\footnotetext{
${ }^{8}$ Ver Titmuss, R., Política Social, Barcelona, Ariel, 1981; Moreno L. y Pérez Yruela, M. (comps.) Política Social y estado del bienestar, Madrid, Ministerio de Asuntos Sociales, 1992.
} 
figura su particular campo conceptual como han podido hacer de forma análoga, por ejemplo, la sociología de las organizaciones, la sociología del deporte o la sociología urbana.

Una comprensión sociológica de la infancia deberá identificar los campos posibles de relación social del/la niño/a y sus distintos problemas sociales considerando siempre que bajo cualquier perspectiva y escuela sociológica el objeto de estudio es el hecho social; es decir, un problema que ha sido producido por la sociedad y cuyas causas se encuentran en esa sociedad, en el conjunto de sus interrelaciones.

El principio sociológico anterior permite avistar ya la enorme complejidad que comporta la construcción sociológica de la infancia a causa de los profundos y acelerados cambios que ha experimentado la sociedad moderna. Si antes el sistema relacional de la infancia identificaba, básicamente, los espacios de socialización familiar y escolar como los más importantes para el devenir adulto del/la niño/a, actualmente las transformaciones del mercado laboral, las recomposiciones y descomposiciones familiares, y la centralidad de los medios de comunicación, en especial de la televisión, han configurado nuevos escenarios que requieren nuevas explicaciones sociológicas y, por lo tanto, la construcción de nuevas categorías explicativas que articulen teóricamente los espacios de relación social de la infancia.

Se añade, finalmente, el discurso jurídico tecnocrático sobre la infancia, al que preferimos en este estudio incluirlo en el discurso sociológico de corte aplicativo ya que es, principalmente, desde esta perspectiva que se generan intervenciones sobre infancia en la actualidad (Convención Internacional sobre los Derechos del Niño, Códigos, etc.) Sin entrar en un análisis crítico sobre esta producción discursiva, lo que se constata es el creciente fenómeno de normativización y regulación jurídica de problemas sociales (los de infancia, seguridad, pobreza, corrupción, etc.) que no han podido ser resueltos.

De acuerdo con este marco general que ya constituye un importante avance en el intento de ampliar la masa crítica de una sociología de la infancia, indudablemente corresponde al ámbito académico la responsabilidad de trabajar - producir conocimiento- la temática de infancia bajo consideraciones epistemológicas -que no pueden ser otras que las de la sociología- y presupuestos conceptuales que den pie a elaboraciones teóricas lo suficientemente rigurosas, elaboraciones que den cuenta de la complejidad de la realidad concreta y que, a su vez, se nutran de esta. Hemos insistido ya lo suficiente sobre la dificultad que 
supone para una sociología de la infancia el problema de la indiferenciación entre la realidad concreta y la realidad pensada.

Quizá, cabría ilustrar con un elemental ejemplo la idea inmediatamente anterior. Cuando se plantea como un tópico investigativo el problema social del embarazo precoz y no se lo define como objeto de estudio o problema sociológico sobrevienen inmediatamente las dificultades investigativas.

Ante todo, la temática propuesta no cuenta con un enunciado de investigación definido y delimitado conceptualmente; ¿qué se va a indagar de ese problema?, ¿qué grupo de mujeres adolescentes va a ser considerado?, ¿cómo incide el nivel de instrucción?, ¿de qué tipo de familia procede?, etc.

Sobre este tema pueden formularse decenas de interrogantes, todas ellas válidas e incluso factibles para levantar un registro inventariado, pero mientras su enunciado no se inscriba en un marco categorial que determine la elaboración de un marco teórico pertinente, la posibilidad de generar pensamiento sobre el tema correrá el riesgo de quedarse en un simple registro o en el nivel de una especulación ensayística sin sustento investigativo.

Cosa muy distinta sería contar con un enunciado que ya denote un uso conceptual de los términos utilizados. Si se plantea, por ejemplo, "Ausencias paternas y maternidad adolescente: el embarazo precoz en jóvenes de 15-17 años en los colegios de Quito", nótese que ya en el enunciado existen categorías (ausencias paternas y maternidad adolescente) sobre las que habrá que trabajar en una dimensión teórica para comprender mejor las distintas posibilidades que puedan presentar la realidad concreta del grupo a investigarse.

Lo realmente interesante y esencial, en términos de evidenciar la importancia de la problematización sociológica como paso ineludible para eficaces intervenciones y producción de políticas sociales, radica en el hecho de que a través de los resultados de la investigación, que servirán como parte del acumulado de la sociología de la infancia, podrá determinarse la conveniencia o no de posibles intervenciones, de la necesidad de utilización de una u otra estrategia, de la adopción de criterios nuevos para enfrentar este problema, etc.

Al contrario, una posible aplicación directa de conceptos sociológicos generales puede generar una complejidad mayor en el problema social concreto y, de modo más obvio, las posibles intervenciones sus- 
tentadas únicamente en el sentido común, puede profundizar y agudizar el problema hasta situaciones insostenibles.

Sin embargo, de todo lo dicho y de una expresa toma de posición por retomar con seriedad cauces más sociológicos para comprender la infancia, quienes se plantean el reto de ir construyendo una sociología de la infancia asumen, simultáneamente, la responsabilidad de procesar críticamente las propuestas y trabajos que se ubiquen en una línea más infantológica y en el terreno de la sociología aplicada, puesto que desde estos tipos de discursividad también se produce información.

Una sociología de la infancia será tal, si es capaz de deconstruir los discursos infantológicos y mostrar las limitaciones de este tipo de discursos, tanto en el orden teórico como en sus posibles efectos.

\section{Política social y políticas sociales para la infancia y adolescencia: la necesidad de una sociología de la infancia.}

Análisis introductorio sobre la evolución conceptual de la política social.

A menudo, se tiende a identificar política social con Estado de bienestar y, con ello, asociar política social con democracia moderna. Sin embargo, autores como Esping-Andersen ${ }^{9}$, sostienen que ya desde la antigüedad se constató la existencia de política social cuando los romanos repartían víveres y ropa a los pobres, y los gobiernos medievales, a través de distintas asociaciones y gremios, construían sus primeros sistemas de asistencia y seguridad social.

De estos estudios referenciales pueden desprenderse, al menos, dos ideas preliminares: a) Estado de bienestar, política social y democracia moderna no son la misma cosa, y b) que puede existir política social sin Estado de bienestar pero no a la inversa.

Estas dos consideraciones merecen un análisis más detenido y detallado que no nos proponemos desarrollar aquí; el propósito es, apenas, dejar planteada la amplitud y complejidad conceptual del campo de la política social y de sus distintas posibilidades de diseño e implementación según el tipo de Estado y sociedad en el que se genere y opere.

${ }^{9}$ Esping-Andersen, G., The Tree Worlds of Welfare Capitalism. Cambridge, Polity Press, 1990. 
Por ello, cuando se propone el estudio de la política social se hace imprescindible precisar el contexto y, de modo específico, el tipo de Estado, el carácter de las relaciones Estado-sociedad y una cuestión fundamental: las relaciones de interdependencia y complementariedad con las otras políticas (económicas, culturales, educativas, etc.)

Desde esta perspectiva, a la vez que se complejiza el debate, se abre posibilidades para la comprensión del carácter y naturaleza de las variantes del Estado y de las tipologías de política social. A lo largo de la historia se aprecia una suerte de correspondencia más o menos directa entre la política social y el tipo de Estado.

A un Estado liberal, por ejemplo, le correspondió básicamente un determinado tipo de política social residual, es decir, un conjunto de acciones desplegadas por las instituciones de bienestar destinadas a compensar aquellas necesidades individuales que no podían ser satisfechas por las dos vías "naturales" en este tipo de Estado: la familia y el mercado. Y, de forma análoga, el modelo conservador y el modelo socialdemócrata de política social pueden comprenderse en un marco de relaciones socio-estatales específicas.

En cuanto a la interdependencia y complementariedad de los distintos tipos de políticas, hoy más que nunca, esta doble relación evidencia la crisis del Estado de bienestar ya que su interdependencia se sustenta en una complementariedad perversa provocada por la absoluta subordinación de la política social por la política económica. Por otra parte, es bastante difícil asumir la gran mayoría de los Estados latinoamericanos haya alcanzado el calificativo de bienestar -cuestionado ya desde sus orígenes- y que un Estado de bienestar sea igual a otro.

En términos teóricos, la política social (producto de la diferenciación funcional creciente entre las dimensiones económica, política, social y cultural y de sus distinciones analíticas para fines de intervención estatal) en el Estado de bienestar de cuño keynesiano funcionaba como un dispositivo de regulación de la dinámica económica y, a la larga, de la misma democracia. Es decir, se orientaba hacia la neutralización de las diferencias económicas por medios sociales expresados en la asistencia, la seguridad social y la subisidiariedad, principalmente.

Con la crisis del Estado de bienestar, agudizada en la década de los 90 por efectos de la mundialización creciente de la economía y sus efectos en las responsabilidades del Estado para con la sociedad, la polí- 
tica social adquiere formas mucho más asistencialistas, compensatorias y focalizadas por los demoledores efectos de la política económica a nivel mundial.

El carácter de la interdependencia entre el conjunto de políticas públicas sufre un notorio desbalance que sitúa a la política social como un agregado de medidas y acciones meramente paliativas en sus distintos campos de intervención. Se trata de una interdependencia asimétri$\mathrm{ca}^{10}$ en la cual la dimensión económica deja de ser regulada por la política social y pública, para dar paso a un proceso inverso en el que la política económica organiza y dictamina el alcance y cauces de la política social en sus distintos sectores, provocando, además, el retorno hacia un particular tipo de política social (modelo conservador, según Titmuss, op. cit.) en el que la caridad pública, cada vez más escasa, se combina y se transforma en responsabilidad social, eufemismo cada vez más utilizado por las fuerzas corporativas anónimas ${ }^{11}$.

Resultado de lo anterior es que la creciente privatización de la sociedad y de lo público, en general, ha determinado que buena parte de la política social, tradicionalmente incluida en la política pública, está trasladándose a instancias de lo privado y, más específicamente, a instancias del mundo empresarial. La política social, en el momento actual, tiende a convertirse en una más de las posibilidades políticas de las corporaciones privadas.

En esa medida, la complementariedad de las políticas se establece mediante indicaciones con arreglo a una mayor visibilidad discursiva de las políticas sociales pero cuyo efecto complementario -entiéndase de regulación- en lo económico es de muy baja intensidad. Se produce una complementariedad perversa en la que la dimensión de lo social sirve para justificar y dejar intocado un modelo de acumulación que se reproduce, entre otros dispositivos, por medio de las políticas económicas.

Estos aspectos constituyen una de las múltiples dimensiones de análisis que habrá que considerar en el caso específico de las políticas

\footnotetext{
${ }^{10}$ Kamerman, S. B., y Kahn, A.J., (comps.) La privatización y el estado benefactor. México, Fondo de Cultura Económica, 1993.

${ }^{11} \mathrm{Se}$ evidencia la emergencia de un proceso de traslado de las obligaciones y responsabilidades estatales, en el campo de la política social para la infancia, hacia empresas multinacionales que asumen programas de ayuda y hacia el llamado "tercer sector" (público no estatal).
} 
sociales de la infancia. Las preguntas sociológicas para posibles y necesarias investigaciones se centran en la indagación sobre las coberturas, sostenibilidad, ayuda a la familia y ayuda al niño, intereses y expectativas, de las empresas que implementan proyectos de asistencia a la infancia mediante modelos de gestión privada o de cogestión (pública-privada).

\section{Aproximación a las políticas sociales en América Latina}

La narrativa de las políticas sociales tienen su anclaje en las ideas, nociones y, más precisamente, en el horizonte de desarrollo planteado en el período de post guerra, en el cual se asume de modo particular en las elites políticas de América Latina la idea de bienestar, generalmente como variable dependiente del crecimiento económico.

La complejidad de este proceso social debe ser comprendida, en primer término, a partir de la emergencia de discursos enmarcados en racionalidades asistencialistas, por una parte, y en la adopción de un modelo de desarrollo sustentado, básicamente, en el crecimiento económico vía industrialización ${ }^{12}$.

Si bien el tema de políticas sociales, en lo que atañe a su diseño y formulación así como a sus posibilidades aplicativas y evaluación, tiene ya un acumulado importante en la región andina, producto del orden de relaciones políticas y económicas post Segunda Guerra Mundial, resulta necesario ubicar la producción de políticas sociales en la sociológica de reproducción de la sociedad y, de modo específico, en el caso ecuatoriano que, por lo demás, no difiere en lo sustancial de los rasgos estructurales comunes a los países andinos.

Una primera y amplia consideración, creemos, debe formulársela en términos geopolíticos; la región andina queda demarcada -no hablamos en términos geográficos- como tal luego de la Segunda Guerra Mundial. La revitalización del modelo fordista de (re) producción social, asentada en la plataforma productiva, comercial y militar hegemónica de los USA, determina un reordenamiento geopolítico del orbe

\footnotetext{
${ }^{12}$ Más específicamente, en la mayoría de países de América Latina se adopta, bajo diversas formas, el modelo de desarrollo industrial sustitutivo de importaciones, lo cual suponía, entre otras transformaciones estructurales del Estado y la sociedad, el paso de sociedades agrarias a sociedades industrializadas.
} 
en el que, por diversas razones de orden sobre todo estratégico y comercial, la compartimentación anterior por continentes resulta bastante insuficiente como para definir el carácter y mecanismos de intercambios comerciales entre los "centros" y "periferias".

Los estados latinoamericanos, sujetos de este orden de relaciones sociales internacionales y en condición de países proveedores de materias primas, inducidos, además, por estrategias de intervención para el desarrollo, plantean el modelo de industrialización por sustitución de importaciones, en el marco del discurso dominante sobre desarrollo.

El ideal, en lo fundamental, era dejar de depender, bajo principios más o menos progresivos de los medios de producción industriales y de las tecnologías como condición básica para el desarrollo, entendido este como niveles de bienestar generalizado y expresado básicamente en indicadores de salud, educación, vivienda, infraestructura, consumo, esperanza de vida, ingresos, estabilidad y promoción laboral, etc.

Esta condición para el desarrollo, validada en las sociedades con procesos industriales ya instaurados en algunos países desde el siglo XIX, fue planteada sin considerar varios presupuestos, entre ellos, el real volumen y magnitud de conocimiento acumulado y expresado en las tecnologías de producción masificada y diversificada. Se desconoció, por ejemplo, que como todo "producto", el conocimiento en cuanto bien socialmente producido, sigue también la lógica de acumulación concentradora de cualquier mercancía. En tal sentido, los países exportadores de materia prima quedaron cada vez más relegados de este fundamental insumo de partida para la producción de medios de producción. En suma, los inevitables requerimientos de medios de producción fueron amplia e intensivamente capitalizados en América Latina por facciones de las burguesías emergentes y tradicionales, directos herederos de las viejas estructuras de reproducción económica y social.

En este escenario general, muy gruesamente delineado, el diseño de políticas sociales nutrió la planificación del desarrollo de los estados de la región, de forma diversa y diferenciada.

En el caso ecuatoriano, donde se lleva ya más de cuatro décadas de diseño y aplicación de políticas sociales, el balance de este conjunto de indicaciones, programas y estrategias es, en términos generales, deficitario. Desde la realidad estadística, la situación de empobrecimiento 
no solo se ha incrementado aceleradamente sino que presenta tendencias agresivas de agudización ${ }^{13}$.

Este resultado plantea una aparente paradoja, puesto que la planificación y planes de desarrollo diseñados por el Estado desde los años 60 fueron calificados de técnicamente bien planteados y proyectados. Incluso, muchas de las indicaciones de política y, específicamente, de política social parecían condensar las aspiraciones y expectativas de la sociedad en su conjunto.

Con el endeudamiento agresivo propiciado en la década del 70, cuando el Ecuador se convierte en país exportador de petróleo, la necesidad de políticas compensatorias que pudieran atenuar los efectos de graves desequilibrios fiscales que determinaron recortes en el gasto público, fue cada vez más notoria desde la lógica de los gobiernos y de los organismos multilaterales de crédito.

A partir de los 80, cuando definitivamente la crisis es inocultable y su estado embrionario se transforma en un incontenible y gigantesco lastre, las políticas sociales empiezan a diseñarse con mayores niveles de sofisticación, pero siempre bajo una lógica de gestión gubernamental que en términos de política social combinaba el modelo residual y el modelo conservador y renunciaba al modelo socialdemócrata de asistencia.

Es en este escenario, en la práctica cooptado por el Estado desarrollista ${ }^{14}$, que se genera un estado de movilización de ciertos sectores de la sociedad, principalmente del movimiento indígena. Se han seguido planteando, en las dos últimas décadas, lineamientos de política social, orientaciones generales de políticas de infancia, e incluso se han activado programas sectoriales cuyos nichos principales constituyen, básicamente la educación y la nutrición de niños en condiciones de pobreza.

Nada de esto, sin embargo, ha detenido el galopante deterioro de las condiciones de vida de niños y adolescentes, que constituyen más de la mitad de la población ecuatoriana. Al contrario, las proyecciones muestran un sombrío futuro especialmente para las más jóvenes generaciones por las tendencias de inversión decreciente en los sectores de salud y educación.

${ }^{13}$ cfr. Informe Económico Cepal, 1982-1999. Diecisiete años de neolioberalismo. 
Si se alude a la necesidad de pensar las políticas sociales de infancia desde la perspectiva de los derechos del niño, la situación se complejiza enormemente al constatar que en el Ecuador, según datos de UNICEF y del Observatorio de los Derechos de la Niñez y Adolescencia, el índice de cumplimiento de los derechos no llega ni a un magro $4 / 10$, considerando todas las edades de infancia y adolescencia.

Todo el conjunto de consideraciones expuestas no son más que vectores de análisis que pretender suscitar indagaciones más específicas en cada uno de los campos de política social (educación, salud, vivienda, bienestar) y en las dimensiones y programas específicos de atención a infancia y adolescencia.

Entre los principales elementos de análisis habrá que considerar el carácter profundamente inequitativo del proceso económico nacional en sus dimensiones de acumulación y redistribución de la riqueza, para lo cual las instituciones que trabajan en el campo de infancia requieren no solo la información sino la explicación sobre el presupuesto general del Estado, sus formas de financiamiento, redistribución y gasto. La explicación de estos aspectos constituye un paso importante en la construcción de una sociología de la infancia y en el intento de ir más allá de trabajar las políticas de infancia en condiciones de creciente preacariedad.

Es en este complejo contexto que la necesidad de pensar una sociología de la infancia y una sociología de las políticas sociales, se hace cada vez más impostergable. En suma, una sociología desprovista de investigación y limitada a la aplicación de principios generales supuestamente válidos para cualquier campo de la problemática social, no solo que postergará posibles transformaciones en la relación sociedad-instituciones-infancia, sino que profundizará las graves problemáticas de la infancia y adolescencia en sus ámbitos familiar, institucional y social.

Esta narrativa de la aplicación de las políticas sociales tiene que ser explicada desde las distintas concepciones sobre el tema de política social, el cual puede condensarse en dos tendencias: aquella concepción de Política Social como agregación de sentidos societales de distinto orden(económico, político, social) en el que de modo explícito "lo social" constituye una dimensión integradora de las prácticas sociales (económicas, políticas, etc...) y, por otra parte, aquella concepción de políticas sociales sustentadas en una racionalidad asistencial y compensatoria, tendencia que se ha venido aplicando en la región. 
La primera de estas tendencias entraña una proyección transformadora en los órdenes político y societal. La segunda opción se orienta hacia la compensación focalizada de los cada vez más perversos efectos producidos por la aplicación de políticas económicas en el actual contexto de capitalismo global.

La primera opción supone, entre otros aspectos, el reconocimiento de la complejidad existente, tanto en el terreno conceptual como en la experiencia institucional en el tratamiento de la política social. Exige, además, revaluar el prestigio de lo social en un mundo en el que se impone el gobierno económico de lo político y, revaluar en suma, lo social como eje y finalidad de una sociedad sujeta a la privatización creciente de lo público como resultado del gobierno de políticas neoliberales.

La segunda posibilidad, es decir la concepción dominante en la producción de las políticas sociales se asienta en presupuestos en los cuales se asume que la sociedad se disuelve en lo económico y en la que sigue subyacente un modelo de desarrollo que, con distintas variantes, se traduce en un cada vez más inquietante e incierto crecimiento económico.

Lo grave del tema en política social es que bajo esta concepción y sus distintos enfoques se interviene en las distintas problemáticas de infancia, hecho que además de propender a aplicaciones artificiosas, a la larga ineficaces y encubridoras de realidades profundas, se legitima indirectamente la "dictadura de lo económico" y la racionalidad de mercado que subordina la racionalidad estatal.

A modo de conclusión general puede decirse que una de las posibilidades de redefinición de la Política Social de Infancia, como instancia de agregación de diversas dinámicas (económica, cultural y política) radica en los aportes que una sociología de la infancia pueda hacer. Las compatibilidades entre la lógica y tiempos de producción intelectual y los tiempos de producción de politicidad deberán encontrar espacios de sinergia. Seguir avanzando en paralelo supone graves riesgos especialmente para los sectores y grupos empobrecidos y mayor probabilidad de re-producción de violencias en un mundo caracterizado por la intensificación de la violencia, cuyas expresiones más brutales y dramáticas se visibilizan, hoy más que nunca, en niños y adolescentes (y no solo de estratos socioeconómicos empobrecidos...). 